Western University

Scholarship@Western

Department of Economics Research Reports

Economics Working Papers Archive

1974

\title{
Individual Welfare, Majority Voting, and the Properties of a Linear Income Tax
}

Thomas Romer

Follow this and additional works at: https://ir.lib.uwo.ca/economicsresrpt

Part of the Economics Commons

Citation of this paper:

Romer, Thomas. "Individual Welfare, Majority Voting, and the Properties of a Linear Income Tax." Department of Economics Research Reports, 7402. London, ON: Department of Economics, University of Western Ontario (1974). 
a

$=$

Research Report 7402

INDIVIDUAL WELFARE, MAJORITY VOTING, AND THE PROPERTIES OF A LINEAR INCOME TAX Fintion: $\cdots$

by

Thomas Romer FES 251974

February 1974 
INDIVIDUAL WELFARE, MAJORITY VOTING, AND THE PROPERTIES OF A LINEAR INCOME TAX ${ }^{*}$

Thomas Romer

University of Western Ontario

Lond on, Canada

\author{
"Pay the income tax \\ And break your heart upon' $t$ " \\ Elizabeth Barrett Browning, \\ Aurora Leigh
}

\title{
I. INTRODUCTION
}

Straightforward suggestions such as Ms. Browning's notwithstanding, the question of choosing parameters of tax functions remains a fundamental problem in public economics. Interest in the theoretical analysis of the political economy of personal income taxation, on the wane for some time, has been reviving lately, due in large part to recent work by Mirrlees (1971). The basic issues center around the following problem: The government is required to raise a certain amount of revenue by taxing the public. How should the tax burden be distributed, given that the imposition of the tax will generally have an impact on individual decisions about work and leisure, on national income, as well as on the distribution of individual incomes and well-being?

Involved in this problem are inevitable conflicts of interest-between social goals and individual we11-being as well as among individuals-whose resolution cannot ultimately be made by economic analysis. Problems regarding the selection of an appropriate social choice criterion to deal with such conflicts lie more properly in the province of the philosophy of justice--the work of Rawls (1971) being a significant example. Economic 
analysis does, nevertheless, have an important role to play in this area. The acceptability of any particular criterion of social choice must depend, at least in part, on its economic implications.

It is in this spirit that we use a descriptive model to examine the nature of the conflicts of interest that may arise in choosing the parameters of a simple type of income tax. Our basic model is similar to those in Atkinson (1973, 1974), Mirrlees (1971), and Sheshinski (1972), but allows for the possibility of a positive revenue requirement by the government. The tax function under consideration is one that is Iinear in before-tax income and includes the possibility of lump-sum grants or levies. Such a tax function has been the subject of considerable policy interest due to the currency in many Western countries of numerous "negative income tax" proposals.

In Section II, we describe the basic model, paying particular attention to the tax-parameter alternatives available to the government. In Section III, this investigation is pursued in greater detail. After a discussion of the behaviour of individual preferences, Sections IV and V focus on the distributional implications of using majority voting to select the parameters of the income tax function.

\section{THE MODEL}

Individual Behaviour

Each individual is assumed to be characterized by a non-negative "index of ability", $n$, which can equally be taken as a measure of his "human capital" or labour productivity. An individual with ability $n$ works a fraction $L_{n}$ of a unit time period, during which he earns an income before tax given by 


$$
z_{n}=n_{n} .
$$

He pays an amount $T\left(z_{n}\right)$ in income tax. Since we are dealing with a static case, it is assumed that the individual consumes all of his after-tax income $y$, so his consumption is given by

$$
C=y=z-T(z) \quad \text { i.e., } C=n L-T(n L) \text {. }
$$

The individual chooses the amount he works and consumes by maximizing a function $U(C, L)$ subject to the budget constraint. The function $U(C, L)$ is assumed to be the same for every member of the society; in fact we will take it to be of Cobb-Douglas form, namely:

$$
U(C, L)=C^{\alpha}(1-L)^{\beta} \text { with } 0<\alpha<1 \text { and } 0<\beta<1 \text {. }
$$

It will be seen that $U_{1}(0, L)=\infty$ and $U_{2}(C, 1)=\infty$ so that zero-consumption and zero-leisure choices will be avoided.

The tax function is assumed to be linear in before-tax income:

$$
T(z)=k+t z \text {. }
$$

We shall refer to $k$ as the lump-sum parameter. If $k$ were to have a negative value, (1) would correspond to the much-proposed negative income taxes of Friedman, Tobin and George McGovern. Those who did not work would then have an after-tax income (i.e., consumption) equal to $-k$.

With these assumptions, we can speak of the individual (C, L) choices in terms of the individual's ability level n. (Following Mirrlees (1971), we can speak of "n-man's" decisions.) From individual maximization, the before-tax income of $n$-man is seen to be dependent on the tax structure:

$$
z_{n}(k, t)= \begin{cases}0 & \text { for } n<m \\ \frac{n(1-t)+\gamma k}{(1-t)(1+\gamma)} & \text { for } n \geq m\end{cases}
$$

where we have defined $\gamma \equiv \beta / \alpha$ and $m \equiv \frac{-\gamma k}{1-t}$. We note immediately that the 
marginal tax rate had better be less than unity to ensure positive aggregate income with a non-negative total revenue requirement.

People with ability less than $m$ will choose to be idle. If $n>0$ for all individuals, then for tax functions with $k>0$, everyone will do some work. From (2) we can see that before-tax income is a nondecreasing function of ability level and for $n>m$ it increases with skill, for a given pair of tax parameters.

In order to explore individual preferences among alternative pairs of tax parameters, it will be useful to express the individual's maximized utility in terms of the tax parameters. To do so, we define the indirect utility function $V_{n}(k, t) \equiv U\left[C_{n}^{*}, L_{n}^{*}\right]$, where $C_{n}^{*}$ and $L_{n}^{*}$ are n-man's utilitymaximizing consumption and labour choices, respectively, for a given pair of tax parameters. Thus,

$$
v_{n}(k, t)= \begin{cases}(-k)^{\alpha} & \text { for } n<m \\ \frac{\delta[n(1-t)-k]^{\alpha+\beta}}{[n(1-t)]^{\beta}} & \text { for } n \geq m\end{cases}
$$

where $\delta=\frac{\gamma^{\beta}}{(1+\gamma)^{\alpha+\beta}}>0$

\section{The Society}

The fraction of the total population that consists of individuals with ability index equal to $n$ or less is $F(n)$, which can be interpreted as the distribution function of $n$. The lowest value of $n$ is $n_{0} \geq 0$ and the highest is $N$. We assume $F(n)$ to be differentiable and define $\frac{d F(n)}{d n}=f(n) \geq 0$, with $f(n)>0$ for $n_{0} \leq n \leq N$. We also have $\int_{n_{0}}^{N} f(n) d n \equiv \int_{n_{0}}^{N} d F(n)=1$. 
On the assumption of constant returns to scale production, per capita national income (before-tax income), is

$$
z(k, t) \equiv \int_{n_{0}}^{N} z_{n}(k, t) d F(n)=\int_{n_{0}}^{N} n L_{n}(k, t) d F(n)
$$

The government is required to raise, by taxing the population, an amount G (again, this should be interpreted as a per-capita requirement), which we take to be non-negative. ${ }^{1}$ Since we are focusing on the taxation problem, we are not now concerned with the purpose for which this revenue is required. Rather, we are interested in investigating how the level of required revenue influences the choice of tax parameters. The government's revenue needs impose a constraint on the tax function--total taxes must be no less than G. Recall that $\int_{n_{0}}^{N} \mathrm{dF}(n)=1$, so that this constraint may be written as

$$
k+t \int_{n_{0}}^{N} z_{n} d F(n) \geq G
$$

Obviously, the revenue requirement must not exceed national income $\int_{n_{0}}^{N} n L_{n} d F(n)$. Since $0 \leq L_{n}<1$ for all $n$, it follows that $\int_{n_{0}}^{N} n_{n} d F(n)<\bar{n}$, where $\bar{n}$ is the mean level of ability. Hence we must have $G<\bar{n}$. We shall, however, find even more stringent constraints on government revenue.

\section{The Tax Possibility Frontier}

An individual's after-tax income is given by $(1-t) n I_{n}-k$. If tax parameters were chosen so that $k>n(1-t)$, then $n-m a n$ would be forced to have negative consumption, since he would be required to pay a lump-sum tax in excess of his "take-home" pay even if he were to work all day (and night). It seems reasonable to impose a restriction on the set of tax parameters in order to avoid such occurrences. ${ }^{2}$ The simplest way of doing 
this is to require

$$
k \leq \mathrm{n}_{0}(1-t)
$$

This ensures that after-tax income will be non-negative for all individuals. A tax rate $t$ will be said to be permissible if $t<1$ and (5) is satisfied. For a given value of $G,(4)$ defines a set of $(k, t)$ pairs which will yield at least the required revenue. The boundary of this set is given by

$$
k+t \int_{n_{0}}^{N} z_{n}(k, t) d F(n)-G=0
$$

Values of $(k, t)$ satisfying (5) and (6) define the tax possibility frontier (TPF). The TPF uniquely determines $k$ for given values of $G$ and $t$. To see this, note that $\frac{\partial z}{\partial k} \geq 0$ for all $n$. Thus, if (6) is satisfied by $\left(k^{0}, t^{0}\right)$, it cannot also be satisfied by $\left(k^{1}, t^{0}\right)$, where $k^{1} \neq k^{0}$. Given $G$, then, for each permissible value of $t$ there is a unique value of $k$. This result will allow us to treat the set of alternative tax parameters as being essentially one-dimensional for a given level of government revenue requirement. Recall that those with ability less than $m=-\gamma k /(1-t)$ do not work. With (5) holding, we can substitute from individual maximization (2) into (6) the expression for $z_{n}(t, k)$ to get

$$
k+\frac{t}{1+\gamma} \int_{m(k, t)}^{N} \operatorname{ndF}(n)+\frac{\gamma k t}{(1-t)(1+\gamma)} \int_{m(k, t)}^{N} d F(n)-G=0
$$

For ease of notation, we define the following two functions:

$$
H(m) \equiv \int_{m(k, t)}^{N} d F(n)
$$

and

$$
\mu(m) \equiv \int_{m(k, t)}^{N} \operatorname{ndF}(n)
$$

$H(m)$ is the fraction of the population that continues to work when those with $n<m$ have dropped out of the labour force, so that when everyone 
works, $H(m)$ is equal to unity, $\mu(m)$ is a weighted value of the skill levels of those still in the labour force. The expression $\frac{\mu(m)}{H(m)}$ gives the (truncated) mean ability of those still working. Again, note that when everyone works, $\mu(m)=\bar{n}$, the mean skill level of all individuals. Using these definitions, the TPF may be written:

$$
k+\frac{t_{\mu}(m)}{1+\gamma}+\frac{\gamma k t H(m)}{(1-t)(1+\gamma)}-G=0
$$

\section{THE CASE WHEN EVERYONE WORKS}

Properties of the TPF

It is of some interest to examine the properties of the TPF in more detail, since it exhibits the tradeoff between marginal tax rate and lump sum tax (or subsidy), and sheds light on the limitations imposed on the parameters of the linear tax. In order to do so, we will first consider the case in which the set of tax parameters is restricted in such a way that, for a given revenue requirement, no one would stop working. We choose this case primarily because it provides considerable information with much less analytical complication than does the more general case, which is discussed briefly in Section $\mathrm{V}$.

When the values of the tax parameters $(k, t)$ are such that $m<n_{0}$, everyone will choose to work. That is, there will be a portion of the TPF along which $\mathrm{H}(\mathrm{m})=1$ and $\mu(\mathrm{m})=\overline{\mathrm{n}}$. For this segment, equation (7) becomes simply:

$$
k=\frac{(1-t)[1+\gamma) G-t \bar{n}]}{1+\gamma-t}
$$

Equation (8) gives the TPF provided that the non-negative disposable income requirement (5) is satisfied and that $k>-n_{0}(1-t) / \gamma$, so that no one drops out of the labour force. The first constraint together with (8) yields 


$$
1>t>\frac{(1+\gamma)\left(G-n_{0}\right)}{\bar{n}-n_{0}} \text {, }
$$

while the condition necessary for everyone to work $\left(n_{0}>m\right.$ ) requires that we have

$$
t<\frac{(1+\gamma)\left(\gamma G+n_{0}\right)}{\gamma \bar{n}+n_{0}} .
$$

In order that the TPF as given by (8) contain any points with $t<1$, it had better be true, therefore, that

$$
G<\frac{\bar{n}+\gamma n_{0}}{1+\gamma}
$$

We note first of all that $k \gtreqless 0$ as $t \lessgtr(1+\gamma) G / \bar{n}$, so that a negative value of the lump-sum parameter can be feasible only for a sufficiently large marginal tax rate. If the govermment's revenue requirement is high, the possibility of a demogrant may be ruled out. This would occur if $G>\bar{n} /(1+\gamma)$. (Of course, (10) must still hold--otherwise no linear tax function would exist that could yield the necessary revenue,)

The slope of the TPF (8) is given by

$$
\begin{aligned}
\left.\frac{d k}{d t}\right|_{T P F}=-\frac{\gamma[(1+\gamma) G-t \bar{n}]+(1-t)(1+\gamma-t) \bar{n}}{(1+\gamma-t)^{2}} \\
\text { and }\left.\quad \frac{d^{2} k}{d t^{2}}\right|_{T P F}=\frac{2 \gamma(1+\gamma)(\bar{n}-G)}{(1+\gamma-t)^{3}}>0
\end{aligned}
$$

Let $\mathfrak{E}$ be the value of $t$ for which $k=n_{0}(1-t)$ and call this value the lowest permissible marginal tax rate. It may readily be verified that the TPF is downward sloping in the neighbourhood of $\tilde{t}$. That is, for sufficiently low marginal tax rates, as the marginal tax rate is increased, total tax revenue rises enough to allow a decrease in the lump-sum parameter. Due to taxation's 
disincentive effects on work, however, this trade-off between higher marginal tax rate and lower lump-sum tax (or larger subsidy, if $\mathrm{k}<0$ ) becomes less and less possible, particularly in the region where $t$ is sufficiently high to permit $k<0$. As the marginal tax rate and lump-sum subsidy increase together, individuals contract their hours of work until, even with increased marginal tax rates, it may no longer be possible to have increased lump-sum grants. ${ }^{3}$ In this case, there would be a critical marginal tax rate $\tau$ above which the TPF would be upward sloping.

If the TPF has a turning point within the restricted range of the tax parameters, the value of $\tau$ is given by the solution to

$$
\gamma t-(1-t)(1+\gamma-t)=\gamma(1+\gamma) G / \bar{n},
$$

which is ${ }^{4} \tau=(1+\gamma)-\sqrt{\frac{\gamma(1+\gamma)(\bar{n}-G)}{\bar{n}}}$. For this to occur, of course, it must be true that, at $t=\tau$, even the lowest-skilled persons continue to work. In other words, we require that $m(\tau)<n_{0}$, i.e., that

$$
n_{0}>\sqrt{\gamma(1+\gamma)(\bar{n}-G) \bar{n}}-\gamma \bar{n}
$$

This relationship between the minimum level of ability and the mean level will not necessarily hold in general, of course. 5

Figure 1 displays TPF's for the (restricted) range of tax parameters such that everyone works. Note that frontiers corresponding to higher values of G lie above those for lower G. From the requirement that no one have negative consumption--i.e., $t>\frac{(1+\gamma)\left(G-n_{0}\right)}{\bar{n}-n_{0}}--i t$ follows that for $G>n_{0}$ (per capita revenue requirement exceeding the earning capacity of the least skilled individual) only positive marginal tax rates are permissible. 


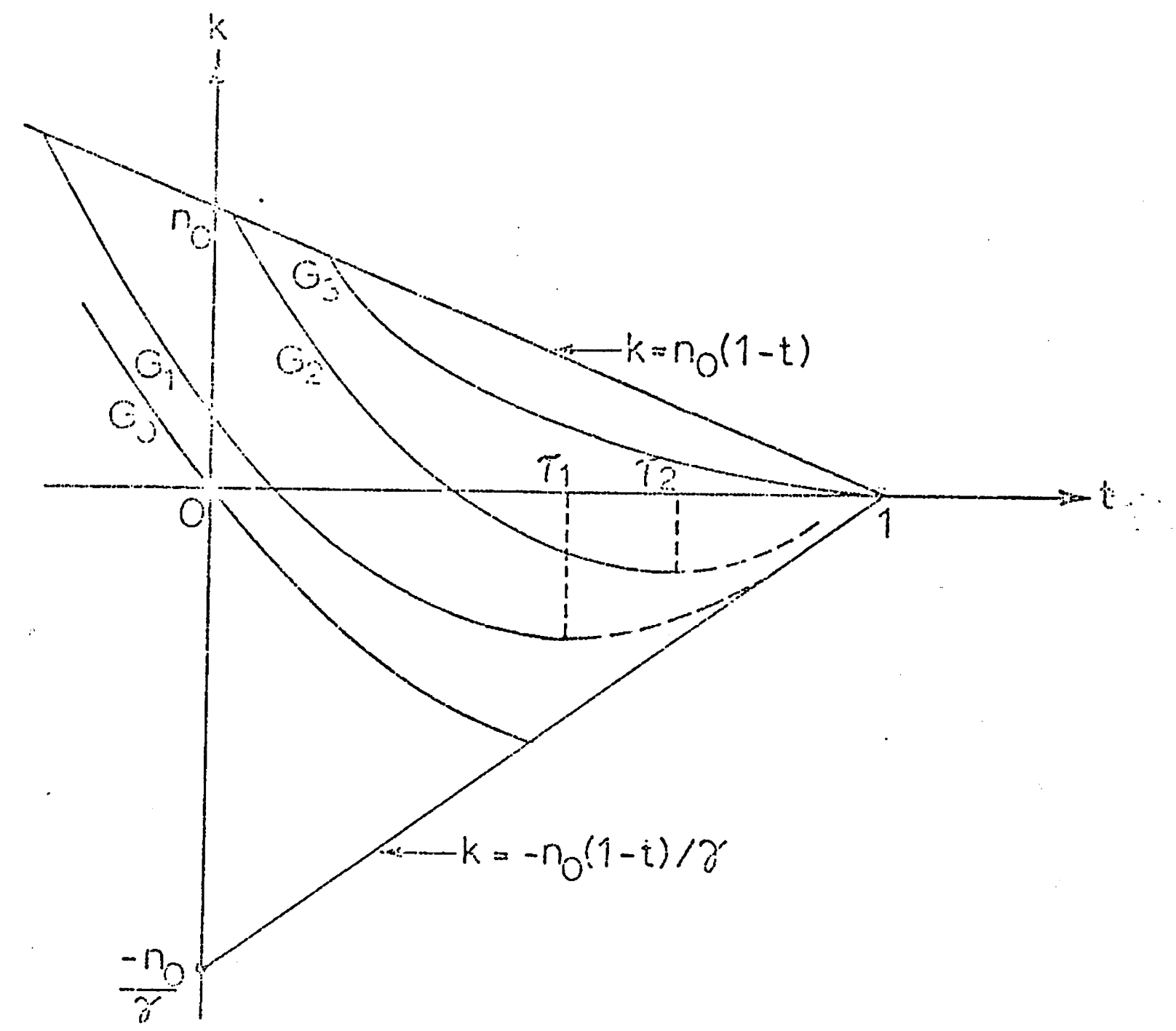

Figure 1. TPFs for various values of $G$, for tax rates such that everyone works $\left(G_{0}<G_{1}<G_{2}<G_{3}\right)$. 


\section{National Income and its Distribution}

As the tax parameters are varied, individuals will adjust their work-leisure choices, so that per capita income before tax will be changing along the TPF. ${ }^{6}$ The average pre-tax income $\mathrm{Z}$ is thus a function of the tax parameters and, when everyone works, is given by

$$
z(t, k)=\int_{n}^{N}\left[\frac{\gamma k}{(1-t)(1+\gamma)}+\frac{n}{1+\gamma}\right] d F(n)=\frac{\gamma k}{(1-t)(1+\gamma)}+\frac{\bar{n}}{1+\gamma}
$$

so that per capita national income is equal to the before-tax income of the worker with average ability. Substitution from (8) gives

$$
Z=\frac{\bar{n}(1-t)+\gamma G}{1+\gamma-t}
$$

and

$$
\frac{d Z}{d t}=\frac{\gamma(G-\bar{n})}{(T+\gamma-t)^{2}}<0 .
$$

Clearly, along the TPF, per capita income before tax (and hence "national incomel) is highest at the lowest permissible value of $t$. In order to attain this maximal level of national income, however, the government would have to set the tax parameters at values such that the least productive individuals would be left with zero after-tax income. ${ }^{7}$ Naturally, such a policy is not likely to be very popular in a society where the proportion of individuals with low ability is relatively high. Nevertheless, this result suggests in a rather stark way the possible conflict in this model between a high level of mean income and considerations of distributional equity. We can expect variations in the tax rate to affect the distribution of after-tax incomes. With no one idle, an individual of ability $n$ has after-tax income $y_{n}$ given by

$$
y_{n}(t)=\frac{n(1-t)}{1+\gamma}+\frac{(1-t)[\bar{n} t-(1+\gamma) G]}{(1+\gamma)(1+\gamma-t)} \text {. }
$$


We have already seen that before-tax income increases with skill level for a given pair of tax parameters. We now observe that, since $\partial y_{n} / \partial n>0$, our tax function has the property that the ranking of incomes is preserved; after-tax income is also increasing with skill level, for a given pair of tax parameters.

Even though the ranking of after-tax income is the same as that of before-tax income, the imposition of the tax will affect the distribution of income. The tax $T_{n}$ paid by $n-m a n$ is

$$
T_{n}(t)=G+\frac{t(n-\bar{n})}{1+\gamma}
$$

so that the "mean individual" (one with skill $\overline{\mathrm{n}}$ ) has a tax burden equal to G regardless of the tax rate--he therefore will not only have before-tax income equal to per capita national income; his take-home pay will be equal to society's average after-tax income. An increase in the marginal tax rate increases (decreases) the tax liability of those with higher (lower) than average ability.

\section{VOTING: THE STMPLE CASE}

\section{Single-peakedness of Individual Preferences}

Concern about the distribution of income is generally motivated (at least implicitly) by the feeling that income is a proxy for well-being. It may be argued persuasively that a society ought to concern itself with the distribution of we11-being and not of income, the latter being, after a11, only one of the factors that influence the former. We turn now to the relationship between individual welfare and the choice of tax parameters. In connection with our discussion of majority voting, we shall be particularly interested in noting whether individual preferences are single-peaked functions of the tax rate. 
When everyone works, n-man's utility is given by

$$
v_{n}(k, t)=\frac{\delta[n(1-t)-k]^{\alpha+\beta}}{[n(1-t)]^{\beta}} ;
$$

from which it follows that, for given permissible values of $(k, t)$, $\partial v / \partial n>0$. This result parallels our finding in the previous section that after-tax income increases with skill level: we see that after-tax utility will also be increasing with ability. The ranking of well-being, as well as that of income, is preserved with the tax under consideration. 8

We now turn to the problem of finding, for an individual with a given skill level, the $(k, t)$ pair that he most prefers out of all those that are permissible. Furthermore, we are interested in discovering whether individuals' preferences over the tax parameters are single-peaked; this information will be useful for our discussion of majority voting in the next section.

Any point on the TPF will lie on some level curve of $V_{n}(k, t)$. This information can then be used to order the points on the TPF according to $n$-man's preferences. Since the TPF is an expression for $k$ in terms of $t$, the problem reduces to one of examining the behaviour of $v_{n}[g(t), t]$ as we change $t$ (where we have written $k=g(t)$ along the TPF).

$$
\frac{d v_{n}}{d t}=\frac{\partial v_{n}}{\partial k} g^{\prime}(t)+\frac{\partial v_{n}}{\partial t}=\frac{\partial v_{n}}{\partial k}\left\{\left.\frac{d k}{d t}\right|_{T P F}-\left.\frac{d k}{d t}\right|_{\bar{V}}\right\}
$$

where $\left.\frac{d k}{d t}\right|_{\bar{V}}$ is the slope of a level curve ${ }^{9}$ of $v_{n}$, evaluated at a point on the TPF; $\left.\frac{d k}{d t}\right|_{T P F}$ is, of course, the slope of the TPF at the same point. since $\partial v_{n} / \partial k<0$, 


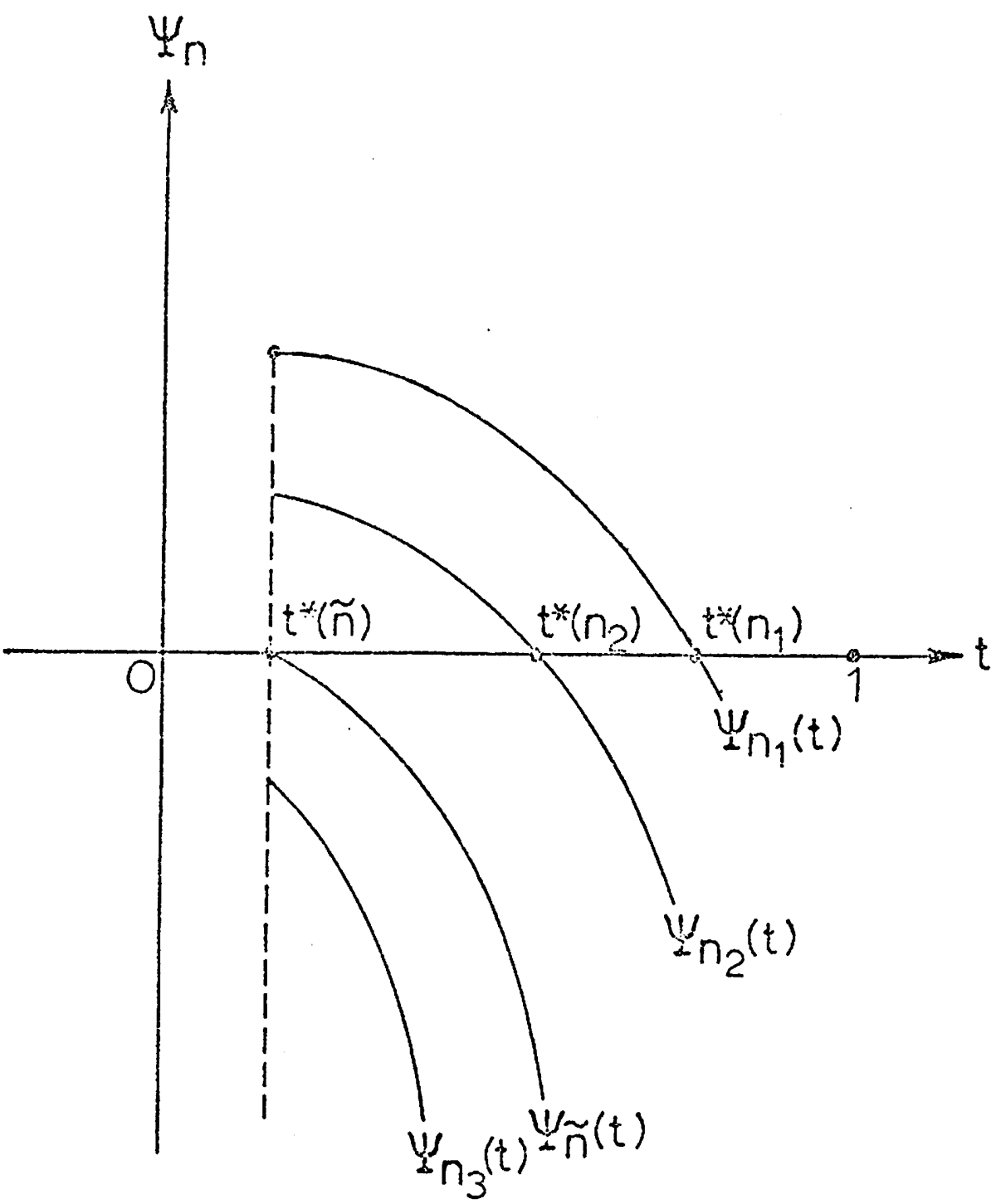

Figure 2. $\Psi_{n}(t)$ functions for various values of $n$.

$$
\mathrm{n}_{1}<\mathrm{n}_{2}<\tilde{\mathrm{n}}<\mathrm{n}_{3}
$$

(Drawn on the assumption that $G>n_{0}$, which implies that only $t>0$ is permissible). 


$$
\operatorname{sign}\left\{\frac{d V_{n}}{d t}\right\}=\operatorname{sign}\left\{\left.\frac{d k}{d t}\right|_{\bar{V}}-\left.\frac{d k}{d t}\right|_{T P F}\right\}
$$

Let

$$
\Psi_{n}(t) \equiv\left\{\left.\frac{d k}{d t}\right|_{V}-\left.\frac{d k}{d t}\right|_{T P F}\right\} \text { along TPF . }
$$

Then

$$
\begin{aligned}
& \Psi_{n}(t)=\frac{\bar{n}-n}{1+\gamma}-\frac{\gamma t(\bar{n}-G)}{(1+\gamma-t)^{2}} \\
& \frac{d \Psi_{n}}{d t}=-\frac{\gamma(\bar{n}-G)(1+\gamma+t)}{(1+\gamma-t)^{3}},
\end{aligned}
$$

so that $\frac{\mathrm{d} \Psi \mathrm{n}}{\mathrm{d} t} \gtreqless 0$ as $\mathrm{t} \lessgtr-(1+\gamma)$; also $\frac{\mathrm{d}^{2} \Psi_{\mathrm{n}}}{\mathrm{dt}^{2}}<0$ if $\mathrm{t}>-2(1+\gamma)$.

We see that for sufficiently high $t--$ and particularly for $t>0--$ $\Psi_{n}(t)$ is a downward-sloping, monotonic function. (See Figure 2). Therefore $\Psi_{n}(t)$ changes sign at most once; it may go from positive to negative but not from negative to positive. Since $\frac{d V_{\mathfrak{n}}}{d t}$ has the same sign as $\Psi_{n}(t)$, we have the following result: Provided that the lowest permissible tax rate is greater than $-(1+\gamma), V_{n}(g(t), t)$ will be single-peaked in $t$, within the permissible range of $t$.

Most-Preferred Tax Rates

Let the lowest permissible value of $t$ be $\tilde{t}>-(1+\gamma)$. Then two possibilities arise:

(a) $\Psi_{n}(\tilde{t})<0$. In this case, $\Psi_{n}(t)<0$ for $t>\tilde{t}$, so that $v_{n}[g(\tilde{t}), \tilde{t}]>v_{n}[g(t), t]$ for $t>\tilde{t}$; actually, n-man's most preferred value of $t$ is below $\tilde{t}$; however, among permissible values, he "most prefers" $\tilde{t}$.

(b) $\Psi_{n}(\tilde{t}) \geq 0$. In this case, there will be a marginal tax rate $t * z \tilde{t}$ such that $V_{n}$ increases from $\tilde{t}$ to $t *$, and decreases for $t>t *$; the most preferred value of $t$ is then obviously $t * .10$ 
Whether case (a) or (b) applies for a given individual will depend on the sign of $\Psi_{n}(\tilde{t})$. From (13)

$$
\frac{\partial \Psi_{n}(t)}{\partial n}=-\frac{1}{1+\gamma}<0
$$

Let the value of $n$ for which $\Psi_{n}(\tilde{t})=0$ be $\tilde{n}$. Thus, there exists a critical level of ability $\tilde{n}$ such that individuals with greater than this ability prefer the lowest permissible tax rate. Those with lower ability prefer some higher tax rate $t *$ given by $\Psi_{n}\left(t^{*}\right)=0$.

The value of $\tilde{\mathrm{n}}$ is readily obtained by setting

$$
\Psi_{\tilde{\mathfrak{n}}}(\tilde{\mathrm{t}})=\frac{\overline{\mathrm{n}}-\tilde{\mathrm{n}}}{1+\gamma}-\frac{\gamma \tilde{\mathrm{t}}(\tilde{\mathrm{n}}-G)}{(1+\gamma-\tilde{t})^{2}}=0,
$$

and substituting $\tilde{t}=\frac{(1+\gamma)\left(G-n_{0}\right)}{\bar{n}-n_{0}}$. This gives

$$
\tilde{n}=\bar{n}-\frac{\gamma\left(\bar{n}-n_{0}\right)\left(G-n_{o}\right)}{\bar{n}-G}
$$

If $G \geq n_{0}$ (so that only non-negative tax rates were permissible), we see that $\tilde{\mathrm{n}} \leq \overline{\mathrm{n}}$.

For $\mathrm{n} \leq \tilde{\mathrm{n}}$, the individual will "most prefer" the value of $t$ for which $\Psi_{n}(t)=0$; i.e., the value of $t$ defined by ${ }^{11}$

$$
\frac{\gamma t(\bar{n}-G)}{(1+\gamma-t)^{2}}=\frac{\bar{n}-n}{1+\gamma}
$$

Equation (14) can be used to define a function $t *(n)$, which gives the value of $t$ most preferred by an individual of ability $n$ :

$$
t *(n)= \begin{cases}\text { solution to (15) } & \text { for } n \leq \tilde{n} \\ \frac{(1+\gamma)\left(G-n_{0}\right)}{\bar{n}-n_{0}} & \text { for } n>\tilde{n}\end{cases}
$$




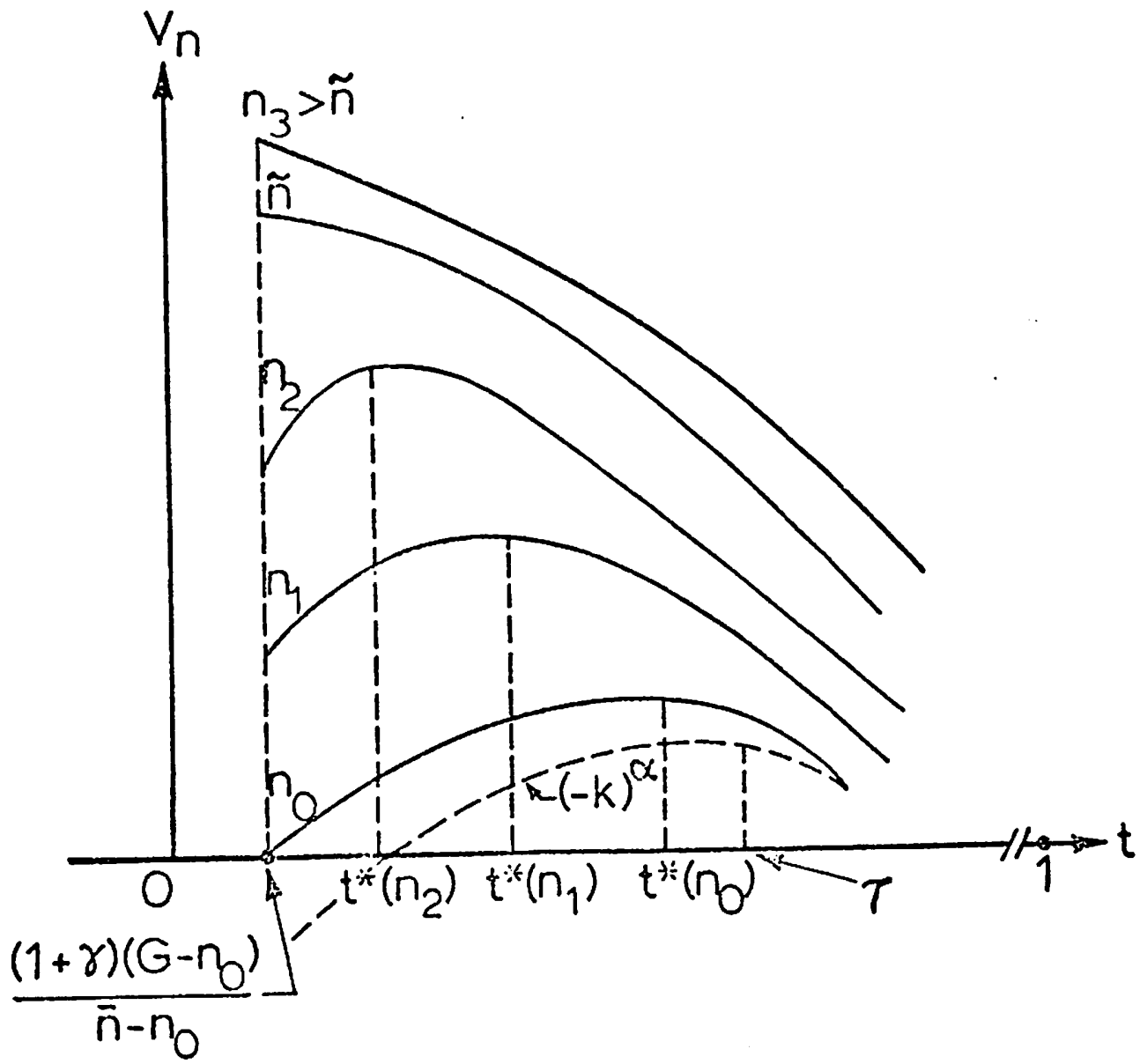

Figure 3. Ranking of tax rates by individuals of various abilities, $\mathrm{n}_{\mathrm{o}}<\mathrm{n}_{1}<\mathrm{n}_{2}<\tilde{\mathrm{n}}<\mathrm{n}_{3}$. 
and $\frac{d t *(n)}{d n} \begin{cases}<0 & \text { for } n \leq \tilde{n} \\ =0 & \text { for } n>\tilde{n}\end{cases}$

Less skilled individuals will have a higher $t *(n)$ than those with greater ability. (See Figure 3).

\section{Majority Voting}

Because the level of individual well-being depends so closely on the tax rate, our analysis shows clearly the kind of conflict involved in choosing particular values for $t$ and $k$. Naturally, were each individual asked to pick his most-preferred value of $t$, he would select the value that makes him best off, i.e., $t *(n)$, in this individualistic world which we have assumed to be devoid of any direct manifestations of altruism or envy. Save in a society composed of identical individuals, however, it will be impossible for a tax rate to be chosen so that it is everyone's "favourite" $\operatorname{tax}_{.12}$ No matter what social choice criterion is used to pick the tax rate, there will be members of society who would prefer some other choice.

Since majority voting is a we1l-known and fundamental aspect of decision-making in democratic societies, it is of some interest to examine the results of using this kind of social choice criterion to determine the tax parameters. In what follows we will suppose that every individual in the society receives exactly one vote and that each voter knows the permissible and possible tax parameters. (In effect, voters know the trade-off between $t$ and $k$ as given by the TPF.) A choice of $t$, uniquely determining $k$, will therefore exactly specify the income tax function.

The single-peakedness of preferences over the permissible range of tax parameters assures us that, in fact, there will be a value of $t$ 
that is stable against the rule of the majority of individuals. That is, there will exist a value of $t--$ call it $\hat{t}$--such that no value of $t$ other than $\hat{t}$ (within the permissible set) is preferred to $\hat{t}$ by strictly more than half the voters. The tax rate $\hat{t}$ is then said to be stable against the rule of majority, since it cannot be defeated by a majority vote in competition with any other permissible tax rate. Furthermore, it is easy to show in this case that $\hat{t}=t *(\hat{n})$, where $\hat{n}$ is the median ability leve1. 13

We would generally expect the median ability to be greater than the lowest ability. As a result, the majority-voting equilibrium (MVE) tax rate will be lower than the tax rate we would pick were we using a Rawlsian maxi-min criterion, since for $\hat{\mathrm{n}}>\mathrm{n}_{0}, t *(\hat{\mathrm{n}})<t *\left(\mathrm{n}_{0}\right)$.

We have seen that for skill levels greater than some critical value $\tilde{n}$, the voter's preferred value of $t$ will be the lowest permissible one, $\tilde{t}$. From equation (14), it will be noted that this critical skill level depends on parameters of the skill distribution ( $\bar{n}$ and $\left.n_{0}\right)$ and on the amount of revenue required, G.

If a majority of the population have skill level greater than the critical value $(\hat{\mathrm{n}}>\tilde{\mathrm{n}})$, then the majority-voting equilibrium tax rate will be the lowest permissible value of $t--i . e ., \hat{t}=\tilde{t}$. In this case, the voting equilibrium will result in the choice of a tax rate which, of all permissible tax rates, minimizes the well-being of the "poorest" individuals, that is those with skill level $\mathrm{n}_{\mathrm{o}}$ :

$$
\left.v_{n_{0}}\right|_{t=\hat{t}} \leq\left. v_{n_{0}}\right|_{\text {a11 permissible } t \neq \hat{t} .}
$$

If, in addition, $G>n_{0}>0$, then the MVE value of $t$ will imply a tax function such that the value of $k$ is positive. ${ }^{14}$ In other words, the tax function 
selected by majority voting will be regressive, in the sense that individuals with low pre-tax incomes will face a higher average tax rate than will individuals with greater pre-tax incomes.

If the skill distribution is skewed rightward (i.e., toward high skill levels), the median skill level will 1je below the mean skill level: $\hat{\mathrm{n}}$ will be less than $\overline{\mathrm{n}}$. Even in this case, which we may take to correspond to much of the "real world", the above result cannot be ruled out. We have found that for the not unreasonable case where $G>n_{0}$, the critical skill value $\tilde{\mathrm{n}}$ would be $\underline{\text { lower }}$ than the mean skill level $\overline{\mathrm{n}}$. It is quite possible, therefore, that we should have $\bar{n}>\hat{n}>\tilde{n}$ and so end up with the MVE described above. Since $\tilde{\mathrm{n}}$ decreases as $\mathrm{G}$ increases, this case is more and more likely to characterize the MVE as we raise the revenue requirement. The MVE need not, of course, be so drastically regressive as to minimize the poorest individual's well-being. Nonetheless, as 1 ong as $t *(\hat{n})<(1+\gamma)(G / \bar{n})$, the tax function selected by majority voting will have a non-negative lump-sum component. In the absence of some form of altruism, therefore, it appears quite possible that even in the case where the majority of voters have incomes below the mean level of income (before tax), the majority-voting equilibrium will result in a regressive income tax function. 15

In order for the MVE tax function to have a "negative tax" component the median skill level must be low enough to have $t *(\hat{n})>(1+\gamma)(G / \bar{n})$. This possibility is ruled out for leftward--i.e., negatively--skewed distributions as long as $G \geq n_{0^{-}}$(To see this, note that $t *(\hat{n})>(1+\gamma)(G / \bar{n})>$ $(1+\gamma)\left(G-n_{0}\right) /\left(\bar{n}-n_{0}\right)=t^{*}(\tilde{n})$ implies that $\tilde{n}>\hat{n}$. But for $G \geq n_{0}$, we have found that $\tilde{\mathrm{n}} \leq \overline{\mathrm{n}}$, while leftward-skewness means $\hat{\mathrm{n}}>\overline{\mathrm{n}}$,) 
V. THE MORE GENERAL CASE

For $t>\frac{(1+\gamma)\left(\gamma G+n_{0}\right)}{\gamma \bar{n}+n_{0}}$ we will have $m>n_{0}$ and individuals with relatively low skill levels sill stop working. In this region, equation (8) no longer holds and we must use the more general expression (7). This causes our problem to become considerably more troublesome. Heuristically, the difficulty lies in the fact that with everyone working, the shape of the TPF and, consequent1y, the behaviour of individual preferences as the tax parameters are varied are independent of the distribution of ability-or, rather, depend only on the (relative) values of $\bar{n}$ and $n_{0}$. When there are people idle, the size of the work force changes as the tax rate changes; and so the behaviour of all of the interesting variables becomes crucially dependent on the entire skill distribution. As a result, we are no longer guaranteed that preferences will be single-peaked for all individuals. In this section, we will first demonstrate that, should preferences be singlepeaked in this more general case, the qualitative results of the previous section will continue to hold. We will then give a sketch of an approach that may be used for checking whether a given skill distribution will yield single-peakedness.

\section{Nationa1 Income}

When the size of the labour force is changing, per capita national income is given by 16

$$
z=\int_{m}^{N} z_{n} d F(n)=\frac{\mu}{1+\gamma}+\frac{\gamma k H}{(1-t)(1+\gamma)}
$$

and as $t$ is varied along the TPF, it can be shown that $\frac{d z}{d t}<0$ (see Appendix), just as in the constant labour force case. The conflict between higher tax rates and mean income remains. 
Voting Results Under Single-Peakedness

Recall that $\frac{d V_{n}}{d t}=-v_{k}\left\{\left.\frac{d k}{d t}\right|_{\bar{V}}-\left.\frac{d k}{d t}\right|_{T P F}\right\}$. In the general case,

$$
\frac{d v_{n}}{d t}= \begin{cases}\left.v_{k} \frac{d k}{d t}\right|_{T P F} & \text { for } n<\frac{-\gamma k}{1-t} \\ -v_{k}\left[-\frac{n}{1+\gamma}-\frac{\gamma k}{(1+\gamma)(1-t)}-\left.\frac{d k}{d t}\right|_{T P F}\right] & \text { for } n \geq \frac{-\gamma k}{1-t}\end{cases}
$$

Hence, $\frac{d V\left(n_{1}\right)}{d t}>\frac{d V\left(n_{2}\right)}{d t}$ for $n_{1}<n_{2}$, unless, of course, both $n_{1}$-man and $n_{2}$-man are idle. This result indicates that if $v_{n}[g(t), t]$ is single-peaked for a11 $n$, then $t *\left(n_{1}\right)>t *\left(n_{2}\right)$ for $n_{1}<n_{2}$, except when both are idle, in which case $t *\left(n_{1}\right)=t *\left(n_{2}\right)$. Consequent $1 y$, the majority voting outcomes would be qualitatively the same as in the case when no one is idle, with the exception that it would now be possible for $t *(\hat{n})$ to have a value such that some individuals would do no work at this tax rate. Choice of a tax rate that immiserizes the poorest persons is still a possible outcome.

Sufficient Conditions for Single-Peakedness

It is shown in the Appendix that if for all m

$$
\operatorname{mf}(\mathrm{m})\left[\frac{\mu(\mathrm{m})-\mathrm{mH}(\mathrm{m})}{\mu(\mathrm{m}) \mathrm{H}(\mathrm{m})}\right]<2
$$

then the shape of the TPF will be such that if its slope is zero for some $t=\tau$, then its slope will be positive for $t>\tau$.

The utility of individuals who are not working is given by $(-k)^{\alpha}$. If condition $(16)$ is satisfied, $(-k)^{\alpha}$ will be a single-peaked function of $t$, so that individuals' preferences will be single-peaked for values of $t$ such that they are idle. Furthermore, it can be shown ${ }^{17}$ that $\frac{\mathrm{dV}_{\mathrm{n}}}{\mathrm{dt}}$ changes 
smoothly as n-man enters or leaves the work force (that is, in the neighbourhood of $\frac{-\gamma k}{1-t}=n$ ). Therefore, if an individual's preferences are singlepeaked for values of $t$ such that he works and if (16) is satisfied, then his preferences will be single-peaked for all permissible tax rates.

We have already established that if the distribution of abilities is such that (12) holds, then everyone will have single-peaked preferences for $t \leq \tau$. Together with (16), this guarantees single-peakedness for all $t<1$ (since for $t>\tau,(16)$ ensures that $k$ and $t$ are both rising, i.e., $V_{n}(k, t)$ is falling for everyone).

If (12) does not hold, the discussion of the previous sections guarantees only that preferences will be single-peaked for tax rates such that everyone works. In this case, (16) ensures single-peakedness only of $v_{n_{0}}$. For $n>n_{0}$, we need a stronger condition. When $n$-man is working, $V_{n}$ will be single-peaked if $\Psi_{n}$ changes sign no more than once. In the constant labour force case, $\Psi_{n}$ is given by (13). More genera11y,

$$
\Psi_{n}=\frac{-\gamma k-n(1-t)}{(1-t)(1+\gamma)}-\left\{\frac{-\gamma k H-(1-t)^{2} \mu}{(1-t)[(1+\gamma)(1-t)+\gamma t H]}\right\} .
$$

In order to derive a condition for single-peakedness that could be applied to the distribution of skills, it is useful to proceed by recasting the problem in terms of the variable m. Now,

$$
m=\frac{-\gamma k}{1-t}, \quad k=G-t z, \quad z=\frac{\mu-m H}{1+\gamma},
$$

so that our transformation becomes

$$
t=\frac{(1+\gamma)(m+\gamma G)}{m[1+\gamma(1-H)]+\gamma \mu}
$$

with $\frac{\mathrm{dt}}{\mathrm{dm}}>0 . .^{18}$ It can then be shown that the sign of $\Psi_{n}$ as $t$ increases behaves the same way as does the sign of the following expression as $m$ increases:

$$
\Phi(m)-(m+\gamma n),
$$


where

$$
\Phi(m)=\frac{\{m[1+\gamma(1-H)]+\gamma \mu\}\{\mu-m H-(1+\gamma) G\}}{\mu-G[1+\gamma(1-H)]}
$$

$\Phi(m)$ will be the same for all workers. A sufficient condition for singlepeakedness of an individual's preferences is that

$$
\Phi(\mathrm{m})=\mathrm{m}+\gamma \mathrm{n}
$$

be satisfied by no more than one value of $m$ for $m \leq n$. Unfortunately, we have not been able to arrive at a satisfactory general a priori restriction on $F(n)$ that would ensure single-peakedness of preferences for all $n$. However, for any particular distribution of skills, $\Phi$ can be derived and the single-peakedness condition checked. If some preferences are not singlepeaked, then there may not exist a majority voting equilibrium tax rate that is stable against all other permissible tax rates. 19

\section{A NUMERICAL EXAMPLE}

As an illustrative example, consider the case when $F(n)$ is the Pareto distribution. Then for $\theta>1$,

$$
f(n)= \begin{cases}\theta n_{0}^{\theta_{n}^{-(\theta+1)}} & n \geq n_{0} \\ 0 & n<n_{0}\end{cases}
$$

and $\frac{\mathfrak{n}_{0}}{\bar{n}}=\frac{(\theta-1)}{\theta}$.

It is easily verified that

$$
\operatorname{mf}(\mathrm{m})\left[\frac{\mu-m H}{\mu \mathrm{H}}\right]= \begin{cases}1 & \mathrm{~m} \geq \mathrm{n}_{0} \\ 0 & \mathrm{~m}<\mathrm{n}_{0}\end{cases}
$$

so that condition (16) will hold for all permissible tax rates. All individuals will then have single-peaked preferences if the relationship between 
the lowest ability level and the mean ability level satisfies (12). This will be the case (everyone will work) if

$$
\frac{\theta-1}{\theta}>\sqrt{\gamma(1+\gamma)(1-G / \bar{n})}-\gamma
$$

Atkinson (1973) has indicated that plausible values of $\theta$ would be between 1.5 and 3.5 and that we would expect $\gamma$ to 1ie between 0.5 and 2.0. For $\theta \geq 2$, inequality (17) is satisfied for all $\gamma$ and $G \geq 0$. Our calculations indicate that for $\theta=1.6$, preferences of individuals close to the mean skill level will not be single-peaked for $G=0$ and $\gamma>1$ but single-peakedness is assured for $G / \overrightarrow{\mathrm{n}}>0.05$ or $\gamma<1$. Further calculations suggest that for $\theta<2$ non-single-peakedness becomes more 1ikely as the dispersion of abilities increases ( $\theta$ decreases), the revenue requirement is close to zero and the relative attractiveness of work is low ( $\gamma$ is high).

In the accompanying tables we have exhibited the values of the majority-voting equilibrium tax rates for $\theta=2$ and $\theta=2.5$ and for various values of $\gamma$ and $G$. We also show, for the sake of comparison, the values of $t *\left(n_{0}\right)$, the tax rate that would emerge if maxi-min were used as the choice criterion. In every case, the difference between $\hat{t}$ and $t *\left(n_{0}\right)$ is substantial, and in several cases the majority-voting tax schedule is regressive while the maxi-min schedule is not. 20

\section{CONCLUSION}

In a society composed of individuals with homogeneous tastes but different earning abilities, variations in the parameters of a linear income tax function have been found to affect the distribution of individual income and welfare as well as the level of aggregate income. Under certain regularity conditions on the distribution of abilities, individual 
Majority voting equilibrium $(\hat{t})$ and maximin $\left(t *\left(n_{0}\right)\right)$ tax rates, Pareto distribution

Table 1. $\quad \theta=2$

\begin{tabular}{|c|c|c|c|c|}
\hline \multirow{2}{*}{$Y$} & & \multicolumn{3}{|c|}{$\mathrm{G} / \overline{\mathbf{n}}$} \\
\hline & & 0 & 0.1 & 0.25 \\
\hline & & .439 & .465 & .510 \\
\hline 0.5 & $\left\{\begin{array}{l}t *\left(n_{0}\right) \\
\text {. }\end{array}\right.$ & .573 & .600 & .647 \\
\hline & $\hat{t}$ & .383 & .411 & $.462 *$ \\
\hline 1.0 & $\left\{t *\left(n_{0}\right)\right.$ & .536 & .569 & .628 \\
\hline & $\hat{t}$ & .358 & .387 & $.441 *$ \\
\hline 1.5 & $\left\{t *\left(n_{0}\right)\right.$ & .522 & .558 & .625 \\
\hline & $\hat{t}$ & .344 & .374 & $.430 *$ \\
\hline 2.0 & $\left\{t *\left(n_{0}\right)\right.$ & .515 & .554 & $.626 *$ \\
\hline
\end{tabular}

Table 2. $\quad \theta=2.5$

\begin{tabular}{|c|c|c|c|}
\hline & \multicolumn{3}{|c|}{$G / \bar{n}$} \\
\hline$Y$ & 0 & 0.1 & 0.25 \\
\hline$r^{2}$ & .363 & .387 & .430 \\
\hline$\left\{t *\left(n_{0}\right)\right.$ & .519 & .546 & .593 \\
\hline & .303 & .327 & $.372 *$ \\
\hline$t *\left(n_{0}\right)$ & .472 & .499 & .559 \\
\hline$\hat{t}$ & .277 & .301 & $.347 *$ \\
\hline$\left\{t *\left(n_{0}\right)\right.$ & .451 & .485 & $.547 *$ \\
\hline$\hat{t}$ & .263 & $.287 *$ & $.333 *$ \\
\hline$\left\{t *\left(n_{0}\right)\right.$ & .440 & .476 & $.541 *$ \\
\hline
\end{tabular}

* indicates that the corresponding value of the lump-sum parameter is positive, i.e., the tax function is regressive in the sense that the average tax rate declines as income increases. 
preferences will be single-peaked with respect to tax rates. For a given government revenue requirement, the poorer individuals tend to favour higher marginal tax rates, while those with greater earning capacity have their well-being maximized at relatively low marginal tax rates. The conflict between high national income and distributional equality is paralleled by a conflict of interest between rich and poor. Majority voting on the tax parameters does not necessarily lead to the adoption of a progressive tax function and may lead to marginal tax rates considerably below those which would be chosen under a Rawlsian maxi-min criterion, even when the distribution of abilities is rightward skew.

In order to make this descriptive analysis reasonably tractable, we have had to make quite strong assumptions about the structure of the problem, especially about the form of individual preferences. At the moment there does not seem to be any truly satisfactory method of getting around this difficulty without imposing other severe restrictions on the model. It would seem to be worthwhile, however, to investigate the sensitivity of our qualitative results to changes in $U(C, L)$. Another important avenue of research would be the development of a satisfactory treatment of the theory of income taxation under heterogeneous preferences. 
FOOTNOTES

*An earlier version of this paper was presented at the Public Economics Workshop held at the University of Essex, Colchester, England, in July 1973. The valuable comments of A. B. Atkinson, A. K. Klevorick, J. E. Stiglitz, and a referee are gratefully acknowledged. A manuscript by $Y$. Itsumi, "Distributional Effects of Linear Income Tax Schedules," dealing with some similar issues, came to my attention after this paper had been completed.

${ }^{1}$ Negative values of $G$ are nevertheless conceivable, as in the cases of imperial booty, foreign aid, oil royalties, etc.

2 Tiberius Caesar was aware of this problem when he observed to his tax-hungry governors, "A good shepherd is supposed to shear his flock, not skin it". ("Boni pastoris esse tondere pecus, non deglubere." ) Perhaps a more natural (and analytically more complex) constraint would be to have $T_{n}=\min \left\{z_{n}-e, k+t_{n}\right\}$, where $e \geq 0$ is a guaranteed subsistence level of consumption.

$$
\frac{\partial L_{n}}{\partial t}=\frac{\gamma k}{n(1-t)^{2}(1+\gamma)}<0 \text { for } k<0 \text { and } \frac{\partial L_{n}}{\partial k}=\frac{\gamma}{n(1-t)(1+\gamma)}>0 \text {; }
$$

i.e., $L_{n}$ decreases as $(-k)$ increases.

${ }^{4}$ Since $G<\bar{n}$, the expression under the square-root is non-negative. Note also that we have rejected the other root, $t>1+\gamma$. If

$G>\bar{n} /(1+\gamma)$, then the TPF is downward-sloping for all permissible values of $t$. In this case, both roots of (11) exceed unity.

${ }^{5}$ Alternatively, we could interpret (12) as a restriction on $\gamma$, as a function of $G, \bar{n}$ and $n_{0}$. For positive $G$, this makes for a very messy expression. In the case of $G=0$, it becomes $\sqrt{\gamma(1+\gamma)}-\gamma<n_{0} / \bar{n}$. (Obvious1y, this cannot hold if $\mathrm{n}_{\mathrm{o}}=0$ )

${ }^{6}$ Population size being fixed, aggregate income will, of course, behave qualitatively the same way as per capita income. After-tax income (per capita) is simply $Z$ - G.

7 If we had used a positive subsistence-consumption value as a constraint instead of (5), this policy would leave the lowest-skilled persons with the subsistence level of after-tax income. 


$$
\frac{{ }^{8} \partial v_{n}}{\partial n}=\frac{\delta[n(1-t)-k]^{\alpha+\beta-1}[\alpha n(1-t)+\beta k]}{n^{\beta+1}(1-t)^{\beta}} \text {. In order for }(k, t) \text { to }
$$

be permissible, it must be true that $k<n(1-t)$ and that $t<1$. Since everyone works, we also have $\frac{-\gamma k}{1-t} \leq n, i . e ., \alpha n(1-t)+\beta k>0$. It follows that $\partial v_{n} / \partial n>0$. From (3) it follows that for those who are idle, we would have $\partial v / \partial n=0$.

The property that well-being increases with ability holds quite generally for all income taxes under homogeneous preferences, not only for the linear case. As an intuitive proof, consider two individuals with different abilities and suppose they both work. Then, under an income tax, the individual with the greater ability can always choose to have the same after-tax income (consumption) as her less-skilled fellow worker. Since she has to put in less working time to earn this income, however, the higher-skilled worker can always end up with higher utility. Only if they both choose not to work will the two of them end up equally well off.

${ }^{9}$ A level curve of $V_{n}(k, t)$ has slope

$$
\left.\frac{d k}{d t}\right|_{V=\bar{V}}= \begin{cases}0 & n<\frac{-\gamma k}{1-t} \\ -\frac{n(1-t)+\gamma k}{(1+\gamma)(1-t)} & n \geq \frac{-\gamma k}{1-t}\end{cases}
$$

Note that when the individual is not working, he is on the flat portion of a level curve, since increases in the value of $t$ alone will not affect his well-being.

${ }^{10}$ If (12) does not hold, there may arise cases (for relatively low $\mathrm{n}$ ) such that $\Psi_{n}(t)>0$ for the highest value of $t$ within the restricted range as defined by (9b). In this case, the individual's "most-preferred" tax rate is taken to be this value $t$.

11 We omit one root which is larger than $(1+\gamma)[1+\gamma(\bar{n}-G) / 2(\bar{n}-n)]>(1+\gamma)$.

${ }^{12}$ In our model, this would occur if everyone had the same ability. clearly, in such a case questions of distribution would not arise.

13 The proposition on single-peakedness is based on a series of theorems in Black (1958) and on results due to Arrow (1963) showing, in effect, that single-peakedness of preferences of the sort discussed here is sufficient for the existence of a (non-empty) core in a majority voting game.

To see that $t *(\hat{n})$ is a majority-voting equilibrium, consider a vote between $t *(\hat{n})$ and some other permissible tax rate $t_{1}>t *(\hat{n})$. The lower tax 
rate $t *(\hat{\mathbf{n}})$ will be clearly preferred by individuals with ability $\mathbf{n} \geq \hat{\mathfrak{n}}$ and only by those individuals. Therefore, the fraction of the population that prefers $t *(\hat{n})$ to $t_{1}$ is $\int_{\hat{n}}^{N} d F(n)$. Since $\int_{\hat{n}}^{N} d F(n)=\frac{1}{2}$, no tax rate higher than $t *(\hat{n})$ could defeat $t^{*}(\hat{n})$ by an absolute majority. By a similar argument it also follows that no permissible tax rate lower than $t *(\hat{n})$ could defeat $t *(\hat{\mathbf{n}})$ by an absolute majority.

${ }^{14}$ From (8) we see that $k>0$ for $t<(1+\gamma) G / \bar{n}$. In the case under consideration,

$$
t=\tilde{t}=\frac{(1+\gamma)\left(G-n_{0}\right)}{\bar{n}-n_{0}} \text {. }
$$

For $G>n_{0}>0$, it follows that, since $G<\bar{n}, \tilde{t}<(1+\gamma) G / \tilde{n}$ and therefore the MVE value of $k$ is positive.

15 Foley (1967), in his analysis of a voting problem with a linear tax function, found that a progressive tax would emerge, since "in most societies a pure majority have incomes below the mean income" [Foley (1967, p. 83)]. He observed, however, that since the poor and the wealthy tend not to be equally enfranchised, in real societies the "effective majority" may end up choosing a regressive tax.

Foley's model had labour perfectly inelastically supplied. In our mode1, we find that even without the disenfranchisement of the poor, the majority-voting equilibrium tax need not be progressive. Because of the (possibly quite strong) disincentives to work caused by taxation, many voters may prefer to choose a tax function that, having a relatively low marginal tax rate, assures a relatively high mean income. In fact, the extreme case of a tax function that "soaks the poor" cannot be ruled out.

16 Note that per capita national income need no longer equal the income of the "mean individual".

17 For details of proofs of statements in this section, see Romer (1974).

18 $\operatorname{sign} \frac{d t}{d m}=\operatorname{sign}\{\mu-G[1+\gamma(1-H)]\}$. We require $G<z$. It follows that $0<\mathrm{mH}<\mu-(1+\gamma) G \leq \mu-G[1+\gamma(1-H)]$.

${ }^{19}$ In the case of non-single peakedness, there will be individuals whose well-being would improve by a move in either direction away from a given tax rate. As a result, the argument of footnote 13 will no longer hold. 
${ }^{20}$ An interesting feature of the numerical results in Tables 1 the possibility that for large revenue requirement, the maxiand 2 is the pos function may be regressive. In these cases, the marginal tax rate required to have lump-sum subsidies is quite high (above $75 \%$ for $G / \bar{n}=0.25$ and $\gamma=2$, for example) and even the poorest individual prefers a lower tax rate. (It should be noted, however, that with

$\theta=2.5$ the earning capacity of the worst-off person is fairly high-one-third the mean skill level.) 


\section{REFERENCES}

Arrow, K. J., 1963, Social choice and individual values, 2nd ed. (Yale University Press, New Haven).

Atkinson, A. B., 1973, How progressive should the income tax be? In: Parkin, M., ed., Essays on modern economics (Longmans, London).

Atkinson, A. B., 1974, Maximin and optimal income taxation, Review of Economic Studies, forthcoming.

Black, D., 1958, The theory of committees and elections (Cambridge University Press, Cambridge).

Foley, D. K., 1967, Resource allocation and the public sector, Yale Economic Essays 7:1, 45-98.

Mirrlees, J. A., 1971, An exploration in the theory of optimum income taxation, Review of Economic Studies 38, 179-208.

Rawls, J., 1971, A theory of justice (Harvard University Press, Cambridge).

Romer, T., 1974, Social choice criteria and the properties of a simple income tax, manuscript.

Sheshinski, E., 1972, The optimal linear income tax, Review of Economic Studies 39, 197-302. 
APPENDIX

1. We wish to show that $\frac{\mathrm{d} Z}{\mathrm{dt}}<0$.

$$
\frac{d Z}{d t}=\frac{\gamma H}{(1+\gamma)(1-t)}\left[\left.\frac{d k}{d t}\right|_{T P F}+\frac{k}{1-t}\right\rfloor
$$

For $k>0$, everyone works and we have already established that $\frac{d z}{d t}<0$ in this case. For $\mathrm{k}<0$,

$$
\left.\frac{\mathrm{dk}}{\mathrm{dt}}\right|_{\mathrm{TPF}}=\frac{-\mathrm{k}}{1-\mathrm{t}} \cdot \frac{\gamma \mathrm{H}}{(1-\mathrm{t})(1+\gamma)+\gamma \mathrm{tH}}-\frac{(1-t) \mu}{(1-t)(1+\gamma)+\gamma \mathrm{tH}}
$$

Since $\frac{(1-t) \mu}{(1-t)(1+\gamma)+\gamma t H}>0$ for $t<1$, it follows that

$$
\left.\frac{d k}{d t}\right|_{T P F}<\frac{-k}{1-t} \cdot \frac{\gamma H}{(1-t)(1+\gamma)+\gamma t H}
$$

Now $\gamma H-[(1-t)(1+\gamma)+\gamma t H]=-(1-t)[1+\gamma(1-H)]<0$

$$
\text { i.e., } \quad \frac{\gamma H}{(1-t)(1+\gamma)+\gamma t H}<1
$$

and therefore $\left.\frac{d k}{d t}\right|_{T P F}<\frac{-k}{1-t}$. It follows that $\frac{d Z}{d t}<0$.

2. We are interested in deriving a condition under which the shape of the TPF will be such that if its slope is zero for some $t=\tau$, then its slope will be positive for $t>\tau$.

The slope of the TPF is given by

$$
\frac{d k}{d t}=\frac{m H-(1-t) \mu}{(1+\gamma)(1-t)+\gamma t H}
$$

The denominator is positive for $t<1$.

Let

$$
S(t)=m H-(1-t) \mu
$$


and $S(\tau)=0$ when $\frac{d k}{d t}=0$.

We are seeking a condition under which $S^{\prime}(T)>0$.

$$
\begin{aligned}
& S^{\prime}(t)=H \frac{d m}{d t}+t \frac{d \mu}{d t}+\mu \\
& \left.\frac{d m}{d t}\right|_{t=\tau}=-\left.\frac{\gamma}{1-t} \frac{d k}{d t}\right|_{t=\tau}+\frac{m}{1-t}=\frac{m(\tau)}{1-\tau}
\end{aligned}
$$

and $\quad \frac{m(T) H(m(T))}{1-\tau}=\mu(\tau)$

so that $S^{\prime}(\tau)=2 \mu(m(\tau))+\left.\tau \frac{d \mu}{d t}\right|_{t=\tau}$

and $\quad S^{\prime}(\tau)>0$ if $\tau \frac{\mathrm{d} \mu / d t}{\mu(m(\tau))}>-2$.

Note that this is a condition on the elasticity of the skill levels of those still working with respect to the tax rate. This can be reformulated as a condition on the skill distribution by noting that

$$
\left.\frac{d \mu}{d t}\right|_{t=T}=-\frac{[m(\tau)]^{2}}{1-\tau} f(m(T))
$$

and

$$
\frac{T}{1-T}=\frac{\mu-\mathrm{mH}}{\mathrm{mH}}
$$

(A.1) then becomes

$$
m(\tau) f(m(\tau))\left\lceil\frac{\mu(m(T))-m(T) H(m(\tau))}{\mu(m(\tau)) H(m(\tau))}\right]<2
$$

which will clearly hold, if for all m,

$$
m f(m)\left[\frac{\mu(m)-m H(m)}{\mu(m) H(m)}\right]<2
$$

(A.2) holds when no one drops out of the labour force, since for $m<n_{0}$, $f(m)=0$. 